\title{
L'influence de la conservation sur le nombre total de germes des échantillons de lait réfrigéré
}

\author{
par \\ R. BOSSUYT \\ Station Laitière de l'Etat, Melle \\ Centre de Recherches Agronomiques de l'Etat, Gand (Belgique)
}

\section{INTRODUCTION}

Il est généralement admis que le nombre total de germes est actuellement le paramètre le plus approprié pour fournir une indication quant à la qualité bactériologique du lait réfrigéré. La détermination du nombre total de germes est d'ailleurs utilisée dans la plupart des pays dans le schéma du paiement à la qualité.

Dans la pratique, les échantillons de lait réfrigéré prélevés à la ferme ne peuvent toutefois pas toujours être examinés aussitôt après leur prélèvement. Une certaine multiplication de micro-organismes peut donc se produire pendant le transport de la ferme au laboratoire, tandis que même au laboratoire il faut veiller à garder la qualité bactériologique des échantillons aussi constante que possible jusqu'au moment où l'on procédera à la détermination du nombre total de germes.

Le plus souvent on tâche d'empêcher la multiplication ultérieure de micro-organismes en conservant l'échantillon de lait suffisamment froid pendant la période qui s'écoule entre son prélèvement et l'exécution de l'analyse à la qualité. Heeschen et al. (1969) ont proposé d'ajouter un mélange d'acide borique et de sorbate à l'échantillon afin de garder sa qualité bactériologique inchangée pendant environ $2 \mathrm{j}$. Ce mélange peut être obtenu sous forme lyophylisée dans le commerce. La poudre est préalablement dosée dans des éprouvettes stériles destinées à la conservation d'environ $10 \mathrm{ml}$ de lait.

La recherche décrite ici a été entreprise pour étudier l'influence exercée sur le nombre total de germes par des conservations de $20 \mathrm{~h}$ et $44 \mathrm{~h}$. L'effet de deux températures de conservation, respectivement $4^{\circ} \mathrm{C}$ et $1^{\circ} \mathrm{C}$, ainsi que l'utilité du mélange d'acide borique et de sorbate ont été vérifiés. 


\section{MATERIEL ET METHODES}

Une première série d'essais a été effectuée avec cent-vingt-sept échantillons de lait réfrigéré provenant de quarante-neuf fermes différentes. L'objectif étant d'opérer autant que possible dans des conditions proches de celles de la pratique, les échantillons provenaient de deux, quatre ou six traites. Ces échantillons ont été transportés au laboratoire dans un bac d'isolation à plaques réfrigérantes et leur nombre total de germes a été déterminé dans les $5 \mathrm{~h}$ suivant le prélèvement, selon la norme de la Fédération Internationale de Laiterie (1958).

Sur chaque échantillon on a prélevé ensuite deux fois $10 \mathrm{ml}$ de lait que l'on a transférés dans deux éprouvettes stériles vides et deux tois $10 \mathrm{ml}$ de lait que l'on a transférés dans deux éprouvettes stériles contenant un mélange lyophylisé d'acide borique et de sorbate. On obtenait ainsi quatre sous-échantillons. Les sous-échantillons sans mélange d'acide borique et de sorbate ont été gardés pendant $44 \mathrm{~h}$ dans des pièces réfrigérées, l'une à $4^{\circ} \mathrm{C}$, l'autre à $1^{\circ} \mathrm{C}$. Un souséchantillon additionné d'acide borique et de sorbate a également été conservé $44 \mathrm{~h}$ à $4^{\circ} \mathrm{C}$; l'autre a été gardé $20 \mathrm{~h}$ à température de chambre, puis $24 \mathrm{~h}$ à $4^{\circ} \mathrm{C}$. Le nombre total de germes des différents sous-échantillons a été déterminé après les $20 \mathrm{~h}$ et les $44 \mathrm{~h}$ de conservation.

Partant des résultats de cette première série d'essais, on a soumis à un test de conservation de $20 \mathrm{~h}$ à $1^{\circ} \mathrm{C}$ un total de trois-centsept échantillons provenant de deux-cent-vingt et une fermes différentes.

La répartition en catégories de qualité adoptée était la suivante : $1^{\mathrm{e}}$ catégorie : moins de 100000 bactéries par $\mathrm{ml} ; 2^{\mathrm{e}}$ catégorie : de 100000 à 250000 bactéries par $\mathrm{ml}$; 3e catégorie : plus de 250000 bactéries par $\mathrm{ml}$. La $1^{\mathrm{e}}$ catégorie est désignée ci-après comme la " plus haute » et la $3^{\mathrm{e}}$ comme la «plus basse ».

\section{RESULTATS}

\subsection{Conservation à $4^{\circ} \mathrm{C}$}

Le tableau 1 montre la répartition des échantillons de lait dans les trois catégories de qualité avant et après $20 \mathrm{~h}$ et $44 \mathrm{~h}$ de conservation. Le tableau 2 indique les changements de catégorie survenus au cours de ces deux périodes de conservation. La figure 1 illustre, sous forme de diagramme par points, l'influence exercée sur le nombre total de germes des échantillons quand ceux-ci étaient conservés $20 \mathrm{~h}$ à $4^{\circ} \mathrm{C}$. 
TABLEAU 1

Classification avant et après la conservation à $4^{\circ} \mathrm{C}$

\begin{tabular}{|c|c|c|c|c|c|c|}
\hline & \multicolumn{2}{|c|}{$1^{\mathrm{e}}$ catégorie } & \multicolumn{2}{|c|}{$2^{\mathrm{e}}$ catégorie } & \multicolumn{2}{|c|}{$3^{\mathrm{e}}$ catégorie } \\
\hline & nombre & p. 100 & nombre & p. 100 & nombre & p. 100 \\
\hline Prélèvement & 64 & 50,4 & 27 & 21,2 & 36 & 28,4 \\
\hline $20 \mathrm{~h}$ & 50 & 39,4 & 24 & 18,9 & 53 & 41,7 \\
\hline $44 \mathrm{~h}$ & 20 & 15,8 & 11 & 8,6 & 96 & 75,6 \\
\hline
\end{tabular}

TABLEAU 2

Changements de catégorie survenus au cours de la conservation à $4^{\circ} \mathrm{C}$

\begin{tabular}{|c|c|c|c|c|c|c|}
\hline & \multicolumn{2}{|c|}{ Catégorie supérieure } & \multicolumn{2}{|c|}{ Catégorie inférieure } & \multicolumn{2}{|c|}{ Même catégorie } \\
\hline & nombre & p. 100 & nombre & p. 100 & nombre & p. 100 \\
\hline $20 \mathrm{~h}$ & 0 & 0 & 26 & 20,5 & 101 & 79,5 \\
\hline $44 \mathrm{~h}$ & 0 & 0 & 69 & 54,3 & 58 & 45,7 \\
\hline
\end{tabular}

TABLEAU 3

Classification avant et après la conservation à $1^{\circ} \mathrm{C} / 20 \mathrm{~h}$

\begin{tabular}{|c|c|c|c|c|c|c|}
\hline & \multicolumn{2}{|c|}{$1^{\mathrm{e}}$ catégorie } & \multicolumn{2}{|c|}{$2^{\mathrm{e}}$ catégorie } & \multicolumn{2}{|c|}{$3^{\mathrm{e}}$ catégorie } \\
\hline & nombre & p. 100 & nombre & p. 100 & nombre & p. 100 \\
\hline Prélèvement & 181 & 59,0 & 48 & 15,6 & 78 & 25,4 \\
\hline Après conservation & 164 & 53,4 & 53 & 17,3 & 90 & 29,3 \\
\hline
\end{tabular}


Nombre total de germes $(20 \mathrm{~h})$

(en milliers/ml de lait)

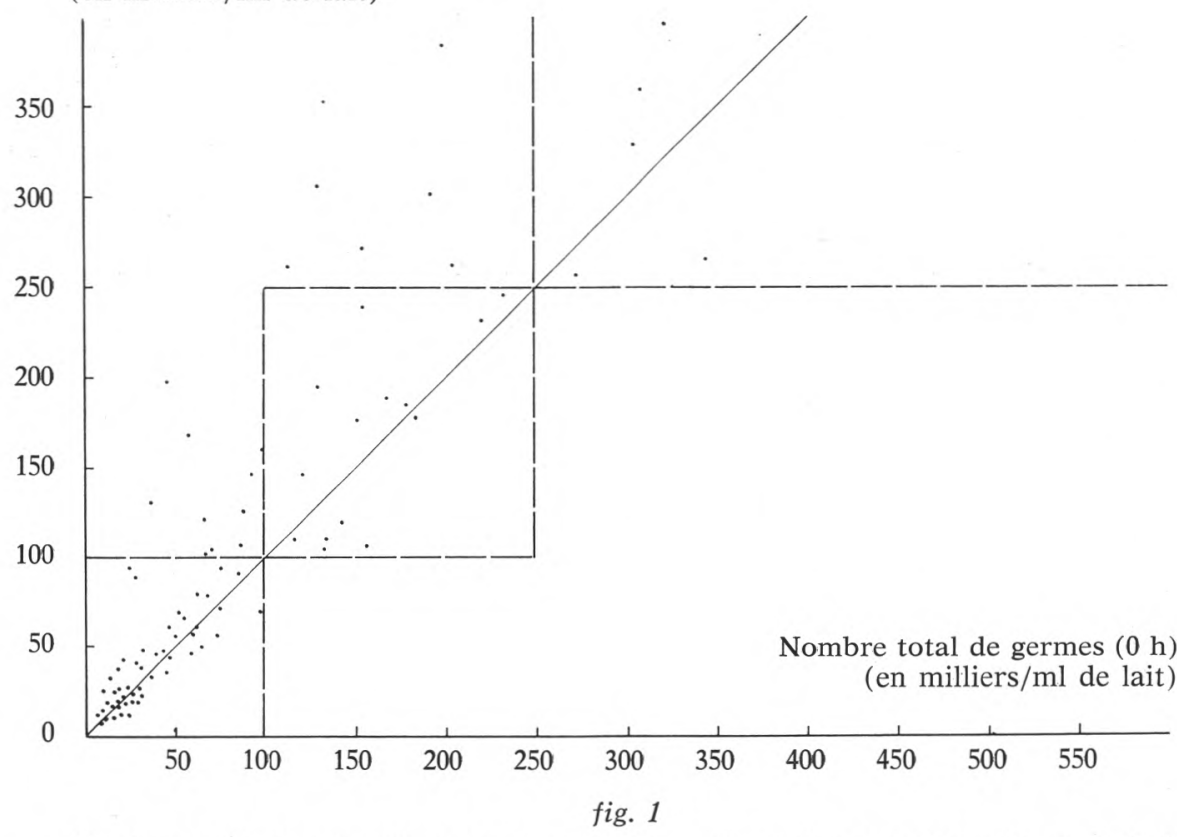

Nombre total de germes avant et après la conservation des échantillons à $4^{\circ} \mathrm{C}$

Nombre total de germes $(20 \mathrm{~h})$

(en milliers/ml de lait)

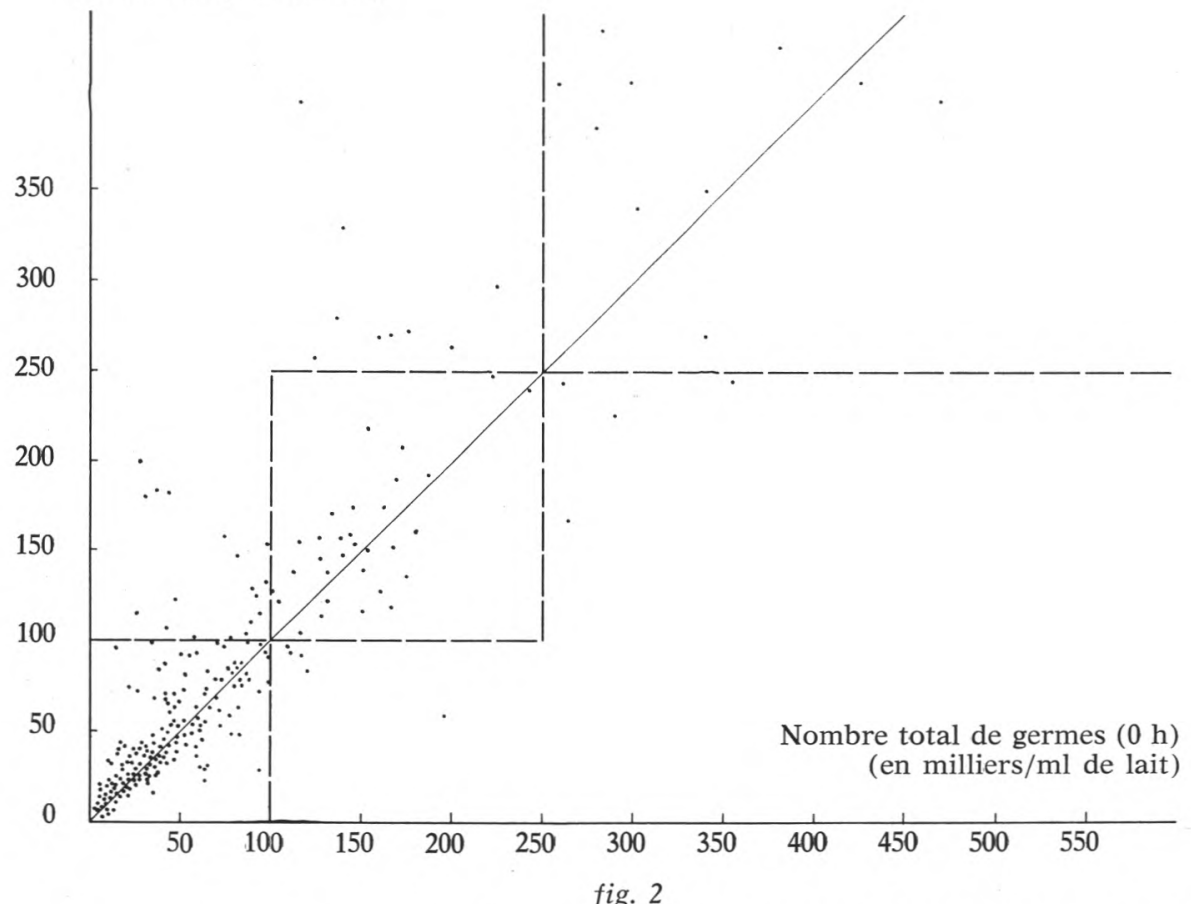

Nombre total de germes avant et après la conservation des échantillons à $1^{\circ} \mathrm{C}$ 


\section{$T A B L E A U 4$}

Changements de catégorie survenus au cours de la conservation à $1^{\circ} \mathrm{C} / 20 \mathrm{~h}$

\begin{tabular}{|c|c|c|c|c|c|c|}
\hline & \multicolumn{2}{|c|}{$\begin{array}{l}\text { Catégorie } \\
\text { supérieure }\end{array}$} & \multicolumn{2}{|c|}{$\begin{array}{l}\text { Catégorie } \\
\text { inférieure }\end{array}$} & \multicolumn{2}{|c|}{$\begin{array}{c}\text { Même } \\
\text { catégorie }\end{array}$} \\
\hline & nombre & p. 100 & nombre & p. 100 & nombre & p. 100 \\
\hline Après conservation & 9 & 2,9 & 34 & 11,1 & 264 & 86,0 \\
\hline
\end{tabular}

TABLEAU 5

Classification avant et après la conservation à $1^{\circ} \mathrm{C} / 44 \mathrm{~h}$

\begin{tabular}{l|c|c|c|c|c|c}
\hline & \multicolumn{2}{|c|}{$1^{\mathrm{e}}$ catégorie } & \multicolumn{2}{|c|}{$2^{\mathrm{e}}$ catégorie } & \multicolumn{2}{|c}{$3^{\mathrm{e}}$ catégorie } \\
\cline { 2 - 6 } & nombre & p. 100 & nombre & p. 100 & nombre & p. 100 \\
\hline & & & & & & \\
\hline & 64 & 50,4 & 27 & 21,2 & 36 & 28,4 \\
Prélèvement & 54 & 42,5 & 19 & 15,0 & 54 & 42,5 \\
Après conservation & & & & & & \\
\hline
\end{tabular}

TABLEAU 6

Changements de catégorie survenus au cours de la conservation à $1^{\circ} \mathrm{C} / 44 \mathrm{~h}$

\begin{tabular}{|c|c|c|c|c|c|c|}
\hline & \multicolumn{2}{|c|}{$\begin{array}{l}\text { Catégorie } \\
\text { supérieure }\end{array}$} & \multicolumn{2}{|c|}{$\begin{array}{l}\text { Catégorie } \\
\text { inférieure }\end{array}$} & \multicolumn{2}{|c|}{$\begin{array}{l}\text { Même } \\
\text { catégorie }\end{array}$} \\
\hline & nombre & p. 100 & nombre & p. 100 & nombre & p. 100 \\
\hline Après conservation & 4 & 3,2 & 31 & 24,4 & 92 & 72,4 \\
\hline
\end{tabular}


On peut déduire du tableau 1 que le nombre d'échantillons de $1^{\mathrm{e}}$ catégorie tombe de 50,4 p. 100 lors du prélèvement à 39,4 p. 100 après $20 \mathrm{~h}$ et à $15,8 \mathrm{p}, 100$ après $44 \mathrm{~h}$ de conservation. Le nombre d'échantillons de $2^{\mathrm{e}}$ catégorie a également diminué. Le nombre d'échantillons de $3^{\text {e }}$ catégorie, par contre, a augmenté de 28,4 p. 100 lors du prélèvement à 41,7 p. 100 après 20 h et à 75,6 p. 100 après 44 h de conservation.

Le tableau 2 et la figure 1 montrent que, dans les conditions de l'essai, après $20 \mathrm{~h}$ de conservation à $4^{\circ} \mathrm{C} 79,5 \mathrm{p} .100 \mathrm{du}$ nombre total d'échantillons ont été classés correctement et 20,5 p. 100 dans une catégorie trop basse. Après $44 \mathrm{~h}$ de conservation à $4^{\circ} \mathrm{C}$ la proportion de classifications correctes n'a été que de 45,7 p. 100 du nombre total d'échantillons et 54,3 p. 100 des échantillons avaient été sousévalués.

\subsection{Conservation à $1^{\circ} \mathrm{C}$}

Le tableau 3 montre comment les échantillons ont été classés avant et après $20 \mathrm{~h}$ de conservation et le tableau 4 indique les changements de catégorie survenus au cours de cette période de conservation. Le tableau 5 reproduit la classification avant et après $44 \mathrm{~h}$ de conservation et le tableau 6 les passages d'une catégorie à une autre enregistrés au cours de la conservation. La figure 2 illustre l'influence exercée sur le nombre total de germes par $20 \mathrm{~h}$ de conservation à $1^{\circ} \mathrm{C}$.

Il ressort des données du tableau 3 qu'une conservation de $20 \mathrm{~h}$ à $1^{\circ} \mathrm{C}$ a fait diminuer de 5,6 p.100 le nombre d'échantillons de la $1^{\mathrm{e}}$ catégorie, tandis que les nombres d'échantillons de la $2^{\mathrm{e}}$ catégorie et de la $3^{\text {e }}$ avaient augmenté de respectivement 1,7 p. 100 et 3,9 p. 100 au bout de la même période.

Le tableau 4 montre qu'après $20 \mathrm{~h}$ de conservation à $1^{\circ} \mathrm{C}$ 11,1 p. 100 des échantillons sont tombés dans une catégorie inférieure, tandis que 2,9 p. 100 sont passés dans une catégorie supérieure et que 86 p. 100 n'ont pas changé de catégorie. Cette dernière constatation se retrouve dans la figure 2.

Comme le montre le tableau 5 , une conservation de $44 \mathrm{~h}$ à $1^{\circ} \mathrm{C}$ diminue de respectivement 7,9 p. 100 et 6,2 p. 100 les nombres d'échantillons des $1^{\mathrm{e}}$ et $2^{\mathrm{e}}$ catégories et augmente de 14,1 p. 100 le nombre d'échantillons de la $3^{\text {e }}$ catégorie. Il ressort du tableau 6 que 72,4 p. 100 des échantillons conservés $44 \mathrm{~h}$ à $1^{\circ} \mathrm{C}$ avaient été classés correctement, 24,4 p. 100 sous-évalués et 3,2 p. 100 surévalués.

\subsection{Conservation au mélange d'acide borique et de sorbate}

Le tableau 7 montre comment les échantillons de lait se répartissaient dans les trois catégories de qualité lors de leur prélèvement, 
TABLEAU 7

Classification avant et après la conservation au mélange d'acide borique et de sorbate

\begin{tabular}{|c|c|c|c|c|c|c|}
\hline & \multicolumn{2}{|c|}{$1^{\mathrm{e}}$ catégorie } & \multicolumn{2}{|c|}{$2^{\mathrm{e}}$ catégorie } & \multicolumn{2}{|c|}{$3^{\text {e }}$ catégorie } \\
\hline & nombre & p. 100 & nombre & p. 100 & nombre & p. 100 \\
\hline Prélèvement & 64 & 50,4 & 27 & 21,2 & 36 & 28,4 \\
\hline $20 \mathrm{~h} / 4^{\circ} \mathrm{C}$ & 69 & 54,3 & 36 & 28,4 & 22 & 17,3 \\
\hline $44 \mathrm{~h} / 4^{\circ} \mathrm{C}$ & 70 & 55,1 & 37 & 29,1 & 20 & 15,8 \\
\hline $20 \mathrm{~h} /$ temp. de chambre & 74 & 58,3 & 28 & 22,0 & 25 & 19,7 \\
\hline $\begin{array}{l}20 \mathrm{~h} / \text { temp. de chambre } \\
24 \mathrm{~h} / 4^{\circ} \mathrm{C}\end{array}$ & 76 & 59,8 & 30 & 23,6 & 21 & 16,6 \\
\hline
\end{tabular}

TABLEAU 8

Changements de catégorie survenus au cours de la conservation au mélange d'acide borique et de sorbate

\begin{tabular}{|c|c|c|c|c|c|c|}
\hline & \multicolumn{2}{|c|}{$\begin{array}{l}\text { Catégorie } \\
\text { supérieure }\end{array}$} & \multicolumn{2}{|c|}{$\begin{array}{l}\text { Catégorie } \\
\text { inférieure }\end{array}$} & \multicolumn{2}{|c|}{$\begin{array}{l}\text { Même } \\
\text { catégorie }\end{array}$} \\
\hline & nombre & p. 100 & nombre & p. 100 & nombre & p. 100 \\
\hline $20 \mathrm{~h} / 4^{\circ} \mathrm{C}$ & 21 & 16,6 & 5 & 3,9 & 101 & 79,5 \\
\hline $44 \mathrm{~h} / 4^{\circ} \mathrm{C}$ & 20 & 15,7 & 3 & 2,4 & 104 & 81,9 \\
\hline $20 \mathrm{~h} /$ temp. de chambre & 22 & 17,3 & 5 & 3,9 & 100 & 78,8 \\
\hline $\begin{array}{l}20 \mathrm{~h} / \text { temp. de chambre } \\
24 \mathrm{~h} / 4^{\circ} \mathrm{C}\end{array}$ & 24 & 18,9 & 2 & 1,6 & 101 & 79,5 \\
\hline
\end{tabular}


Nombre total de germes $(20 \mathrm{~h})$

(en milliers/ml de lait)

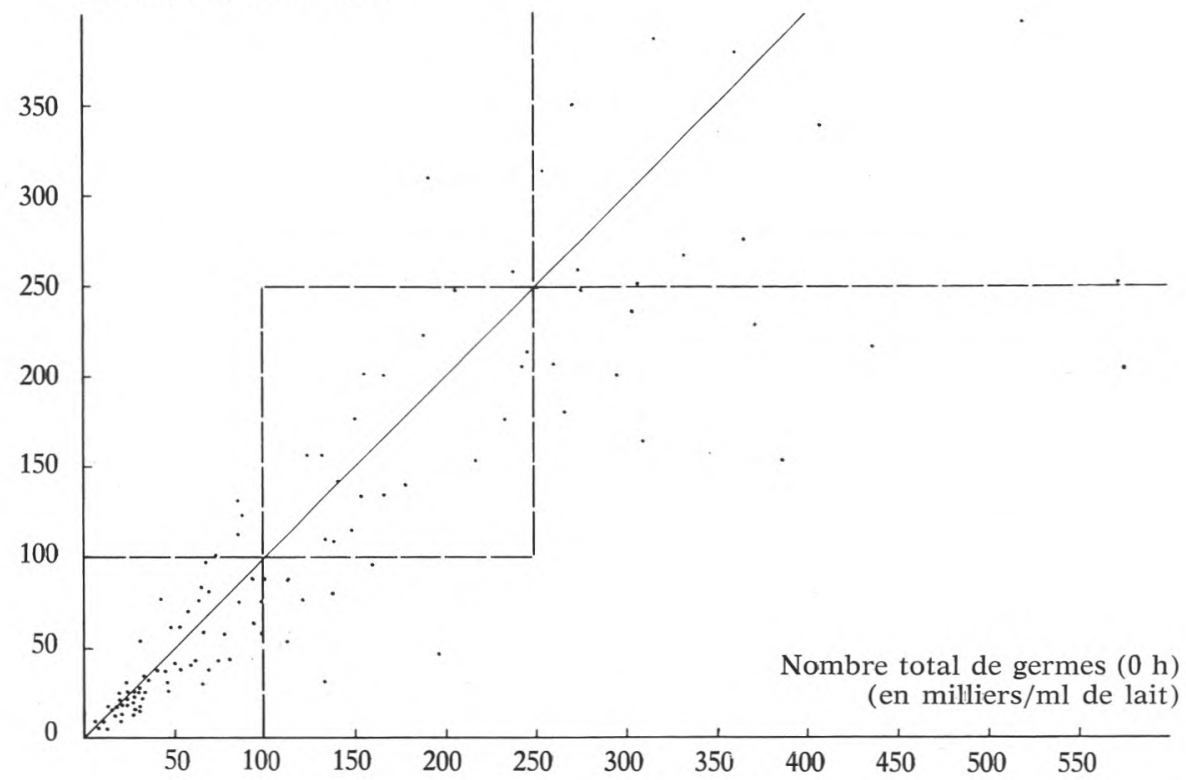

fig. 3

Nombre total de germes avant et après la conservation des échantillons au mélange d'acide borique et de sorbate à $4^{\circ} \mathrm{C}$

Nombre total de germes $(20 \mathrm{~h})$

(en milliers/ml de lait)

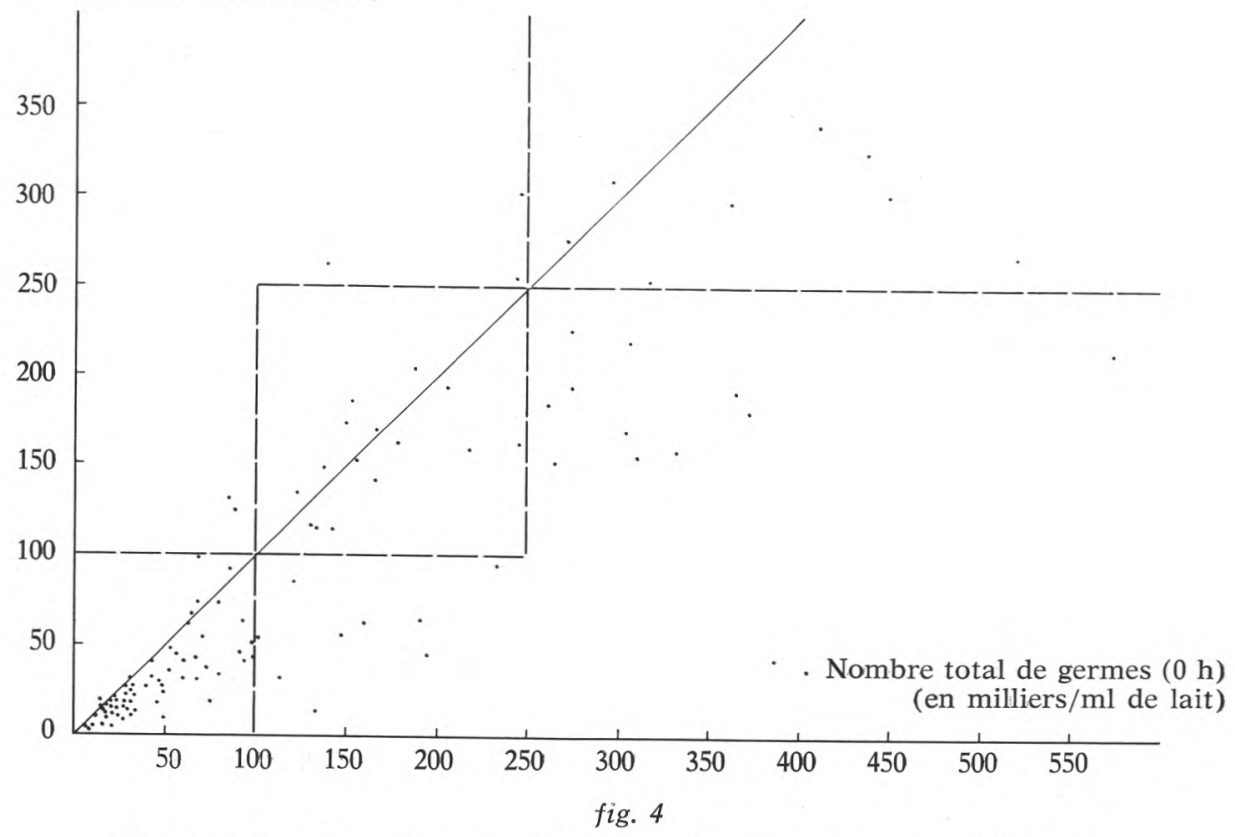

Nombre total de germes avant et après la conservation des échantillons au mélange d'acide borique et de sorbate à température de chambre 
après $20 \mathrm{~h}$ et $44 \mathrm{~h}$ de conservation à $4^{\circ} \mathrm{C}$ et après $20 \mathrm{~h}$ de conservation à température de chambre, puis $24 \mathrm{~h}$ à $4^{\circ} \mathrm{C}$. Le tableau 8 montre les changements de catégorie survenus pendant la conservation. L'influence sur le nombre total de germes de la conservation au mélange d'acide borique et de sorbate, pendant $20 \mathrm{~h}$ respectivement à $4^{\circ} \mathrm{C}$ et à température de chambre, est illustrée sous forme de graphique dans les figures 3 et 4 .

Il ressort du tableau 7 que la durée de conservation n'influe que peu sur la classification finale des échantillons. Aussi bien après $20 \mathrm{~h}$ qu'après $44 \mathrm{~h}$ de conservation à $4^{\circ} \mathrm{C}$ les nombres d'échantillons des $1^{\mathrm{e}}$ et $2^{\mathrm{e}}$ catégories ont augmenté de respectivement 50,4 p. 100 et 21,2 p. 100 lors du prélèvement à respectivement environ 55 p 100 et environ 29 p. 100 , tandis que la proportion d'échantillons de la $3^{\text {e }}$ catégorie tombait de 28,4 p. 100 lors du prélèvement à environ 16 p. 100. Quant aux échantillons conservés d'abord pendant $20 \mathrm{~h}$ à température de chambre, puis pendant $24 \mathrm{~h}$ à $4^{\circ} \mathrm{C}$, on a constaté après les $20 \mathrm{~h}$ aussi bien qu'après les $44 \mathrm{~h}$ que le nombre d'échantillons de la $1^{\mathrm{e}}$ catégorie avait augmenté d'environ 8,5 p.100 et le nombre de ceux de la $2^{\text {e }}$ catégorie d'environ $1,5 \mathrm{p} .100$, tandis que le nombre d'échantillons de la $3^{\text {e }}$ catégorie avait diminué d'environ 10 p. 100 .

Les passages d'une catégorie à une autre survenus pendant la conservation sont à peu près indépendants de la durée de celle-ci. On peut déduire des données du tableau 8 que 81 p. 100 des échantillons restent dans la même catégorie après $20 \mathrm{~h}$ et $44 \mathrm{~h}$ de conservation à $4^{\circ} \mathrm{C}$, tandis qu'environ 16 p. 100 passent dans une catégorie supérieure et 3 p. 100 dans une catégorie inférieure. Après $20 \mathrm{~h}$ de conservation à température de chambre, puis $24 \mathrm{~h}$ à $4^{\circ} \mathrm{C}$, environ 18 p. 100 des échantillons ont passé dans une catégorie plus élevée, environ 79 p. 100 n'ont pas changé de catégorie et environ 3 p. 100 ont passé dans une catégorie plus basse. Les figures 3 et 4 le montrent aussi.

\section{DISCUSSION}

En jugeant ces résultats, il convient de considérer que l'on avait prélevé aussi bien des échantillons de deux que de quatre et de six traites, ce qui veut dire que le lait était parfois gardé depuis déjà $3 \mathrm{j}$ à la ferme, par $4^{\circ} \mathrm{C}$, au moment où l'on prélevait l'échantillon et le soumettait aux différents modes de conservation décrits ci-dessus.

Une conservation de $20 \mathrm{~h}$ à $4^{\circ} \mathrm{C}$ a entraîné une diminution de catégorie pour environ 20 p. 100 d'échantillons ; après 44 h de conservation ceci fut le cas pour environ 54 p. 100 d'échantillons. Il est frappant, sous ce rapport, que lorsque les échantillons contenaient déjà lors du prélèvement un nombre relativement élevé de bactéries (échantillons de $2^{\mathrm{e}}$ catégorie), une température de conservation de $4^{\circ} \mathrm{C}$ n'empêchait pas suffisamment la multiplication ultérieure des 
micro-organismes, de sorte qu'une grande partie de ces échantillons se retrouvait déjà après $20 \mathrm{~h}$ dans une catégorie plus basse. Lorsque le nombre de germes était relativement petit au moment du prélèvement (échantillons de $1^{\mathrm{e}}$ catégorie), le grand déplacement vers les catégories plus basses ne se produisait qu'après $2 \mathrm{j}$ de conservation à $4^{\circ} \mathrm{C}$.

Quoique dans une moindre mesure, le même phénomène s'est produit au cours de la conservation à $1^{\circ} \mathrm{C}$. Ici aussi, une partie des échantillons classés dans la $2^{e}$ catégorie lors du prélèvement n'étaient plus que de $3^{\text {e }}$ catégorie après $20 \mathrm{~h}$ de conservation, tandis que seulement un faible pourcentage d'échantillons de la $1^{\mathrm{c}}$ catégorie est descendu dans une catégorie plus basse. Après $44 \mathrm{~h}$, une partie des échantillons de $1^{\mathrm{e}}$ catégorie a subi le même sort. Ces changements ont toutefois été beaucoup moins prononcés qu'à la température de conservation de $4^{\circ} \mathrm{C}$; après respectivement $20 \mathrm{~h}$ et $44 \mathrm{~h}$ environ 10 p. 100 et environ 25 p. 100 du nombre total d'échantillons se sont retrouvés dans une catégorie inférieure.

Il ressort des essais au mélange d'acide borique et de sorbate que ni la température de conservation $\left(4^{\circ} \mathrm{C}\right.$ ou température de chambre) pendant les $20 \mathrm{~h}$ suivant l'addition de ce mélange, ni la durée de conservation ( $20 \mathrm{~h}$ ou $44 \mathrm{~h}$ ) n'ont influé profondément sur les résultats obtenus. Contrairement aux essais de conservation à $4^{\circ} \mathrm{C}$ et à $1^{\circ} \mathrm{C}$ entrepris sans ce mélange, l'emploi de celui-ci a fait diminuer le nombre total de germes. Le mélange d'acide borique et de sorbate n'est donc pas seulement bactériostatique : il a un certain effet bactéricide. Dans les essais décrits ici, cet effet a résulté en une amélioration de catégorie d'environ 17 p. 100 des échantillons.

\section{CONCLUSION}

On peut conclure de ces essais qu'une conservation des échantillons pendant plus de $20 \mathrm{~h}$ à $4^{\circ} \mathrm{C}$ est à rejeter en tout cas.

Dans les essais décrits ci-dessus, une conservation pendant $20 \mathrm{~h}$ à $4^{\circ} \mathrm{C}$ ou pendant $44 \mathrm{~h}$ à $1^{\circ} \mathrm{C}$ a eu pour conséquence que, respectivement, environ 20 p. 100 et 25 p. 100 des échantillons ont été sousévalués, ce qui représente un pourcentage relativement élevé.

Si l'on emploie le mélange d'acide borique et de sorbate il n'est pas nécessaire de réfrigérer les échantillons pendant les $20 \mathrm{~h}$ qui suivent leur prélèvement (le véhicule d'échantillonnage ne devrait donc plus être équipé d'éléments réfrigérants). Le mélange a toutefois un certain pouvoir bactéricide, de sorte que, dans ces essais, environ 17 p. 100 des échantillons ont été surévalués après une conservation de $44 \mathrm{~h}$. 
Une conservation des échantillons pendant un maximum de $20 \mathrm{~h}$ à $1^{\circ} \mathrm{C}$ semble seule acceptable jusqu'au moment de la détermination du nombre de germes. Dans ces conditions, environ 10 p. 100 d'échantillons ont été sous-évalués au cours des essais. Ce sont principalement des échantillons qui contenaient déjà initialement un nombre de germes relativement élevé (échantillons de $2^{e}$ catégorie), qui sont tombés dans une catégorie plus basse après cette conservation.

A remarquer que les données obtenues ont aussi été exploitées avec d'autres normes de détermination du nombre de germes. Dans ce cas, la $1^{\mathrm{e}}$ catégorie était celle de moins de 200000 bactéries par ml, la $2^{\mathrm{e}}$ celle de 200000 à 500000 bactéries par $\mathrm{ml}$ et la $3^{\mathrm{e}}$ celle de plus de 500000 bactéries par ml. Les résultats n'ont évidemment pas été identiques à ceux décrits dans cette communication, mais la conclusion finale reste valable.

\section{Remerciements}

L'auteur tient à remercier $M$. S. Blockeel, premier ingénieur technique du Groupe de Travail, pour sa précieuse collaboration.

\section{R és u m é}

L'auteur a étudié l'influence exercée sur le nombre total de germes par la conservation pendant $20 \mathrm{~h}$ et pendant $44 \mathrm{~h}$ d'échantillons de lait réfrigéré. Il a vérifié l'effet d'une conservation à $4^{\circ} \mathrm{C}$ et à $1^{\circ} \mathrm{C}$, ainsi que l'utilité d'un mélange d'acide borique et de sorbate.

Une conservation de plus de $20 \mathrm{~h}$ à $4^{\circ} \mathrm{C}$ est à rejeter en tout cas. Selon que les échantillons avaient été conservés $20 \mathrm{~h}$ à $4^{\circ} \mathrm{C}$ ou $44 \mathrm{~h}$ à $1^{\circ} \mathrm{C}$, leur nombre de germes avait tellement augmenté qu'environ 20 p. 100 ou 25 p. 100 , respectivement, des échantillons ont finalement dû être classés dans une catégorie inférieure à celle où ils avaient figuré aussitôt après leur prélèvement.

Le mélange d'acide borique et de sorbate a eu un certain effet bactéricide, qui a résulté en une amélioration de catégorie d'environ 17 p. 100 d'échantillons.

Une conservation pendant un maximum de $20 \mathrm{~h}$ à $1^{\circ} \mathrm{C}$ semble seule acceptable: dans ces conditions, seulement quelque 10 p. 100 des échantillons sont tombés dans une catégorie inférieure parce que des micro-organismes avaient continué de se multiplier pendant la conservation.

Ces résultats doivent être considérés en fonction des normes utilisées pour la classification, en l'occurrence 100000 et 250000 bactéries par $\mathrm{ml}$. La conclusion finale reste toutefois valable si on applique des normes de 200000 et de 500000 bactéries par ml. 


\section{S u m m a r y}

The influence on the total bacterial count of conservation of samples deep-cooled milk for 20 and 44 hours was investigated. Conservation temperatures of $4^{\circ} \mathrm{C}$ and $1^{\circ} \mathrm{C}$ were considered. Besides, the usefulness of a mixture of boric acid and sorbate was tested.

A conservation of samples exceeding 20 hours at $4^{\circ} \mathrm{C}$ should in any case be rejected. A conservation for 20 hours at $4^{\circ} \mathrm{C}$ or for 44 hours at $1^{\circ} \mathrm{C}$ resulted in such an increase in the total bacterial count that approx. 20 p. 100 or approx. 25 p. 100 , respectively, of the samples had to be classified in a class lower than the one they originally belonged to after sampling.

The mixture of boric acid and sorbate had a somewhat bactericidal effect, so that approx. 17 p. 100 of the samples were classified in a higher class.

Only a conservation of samples for maximum 20 hours at $1^{\circ} \mathrm{C}$ seems acceptable; under these conditions only approx. 10 p. 100 of the samples were classified in a lower class, due to further development of microorganisms during that time of conservation.

The standards taken into consideration for the classification were 100,000 and 250,000 bacteria $/ \mathrm{ml}$. The final conclusion is, however, also valid for standards up to 200,000 and 500,000 bacteria $/ \mathrm{ml}$, respectively.

\section{Zusammenfassung}

Der Einfluss einer 20 und 44 Stunden-Lagerung tiefgekühlter Milchproben wurde untersucht. Der Einfluss von Lagertemperaturen von $4^{\circ} \mathrm{C}$ und $1^{\circ} \mathrm{C}$ auf die gesamte Keimzahl und die Brauchbarkeit einer Borsäure-Sorbat-Mischung wurden geprüft.

Eine Probenlagerung, die 20 Stunden bei $4^{\circ} \mathrm{C}$ überschreitet, ist in jedem Fall zu verwerfen. Eine Lagerung während 20 Stunden bei $4^{\circ} \mathrm{C}$ oder während 44 Stunden bei $1^{\circ} \mathrm{C}$ führte $\mathrm{zu}$ einem derartigen Anstieg der gesamten Keimzahl, dass ca. 20 p. 100 bzw. ca. 25 p. 100 der Proben in einer Klasse untergebracht werden musste, die niedriger war als die zu der sie nach der Probenahme gehörten.

Die Borsäure-Sorbat-Mischung war einigermassen bakterizid : ca. 17 p. 100 der Proben konnte in einer höheren Klasse untergebracht werden.

Nur eine Probenlagerung während höchstens 20 Stunden bei $1^{\circ} \mathrm{C}$ ergibt sich als annehmbar; unter diesen Verhältnissen wurde nur ca. 10 p. 100 der Proben in einer niedrigeren Klasse untergebracht, was 
der weiteren Entwicklung von Mikroorganismen während dieser Lagerzeit zuzuschreiben ist.

Die für die Klassifizierung in Betracht gezogenen Normen betrugen 100000 und 250000 Bakterien/ml. Der Schluss gilt jedoch auch für Normen bis 200000 bzw. 500000 Bakterien/ml.

Reçu pour publication en avril 1977.

\section{Références bibliographiques}

Heeschen (W.), Reichmuth (J.), Tolle (A.) und Zeidler (H.) (1969). - Die Konservierung von Milchproben zur bakteriologischen, zytologischen und hemmstoffbiologischen Untersuchung. Milchwissenschaft, 24, 729.

FÉdÉRATION INTERNATIONALE DE LAITERIE (1958). — Numération des micro-organismes du lait liquide et du lait en poudre. Norme Internationale FIL-IDF 3, Bruxelles. 